\title{
Intelligent Maintenance of Moving Joints on Large-scale Machine Tool
}

\author{
Mulan Wang ${ }^{1, a}$, Xuanyu Chen ${ }^{1, b}$, Wenzheng Ding ${ }^{2, c}$, Hao Zhu ${ }^{3, d}$ and Lei Zhu ${ }^{4, ~ e ~}$ \\ ${ }^{1}$ Jiangsu Key Laboratory of Advanced Numerical Control Technology, Nanjing Institute of \\ Technology, Nanjing, China 211167 \\ ${ }^{2}$ Industrial Center, Nanjing Institute of Technology Nanjing, China 211167 \\ ${ }^{3}$ school of Communication Engineering, Nanjing Institute of Technology Nanjing, China 211167 \\ ${ }^{4}$ School of Automation, Nanjing Institute of Technology Nanjing, China 211167 \\ awangml@njit.edu.cn, byjs2004@163.com, cdwz198151@njit.edu.cn, dzhuhao@njit.edu.cn, \\ ezdhxzl@njit.edu.cn
}

Keywords: Intelligent maintenance; Joints; Machine tool; Genetic algorithm (GA), Artificial neural network (ANN).

\begin{abstract}
In order to reduce the maintenance cost and the downtime of large-scale machine tool, the initial weights and thresholds of the back propagation (BP) neural network are optimized to overcome the disadvantages of the slow convergence and fall into the local optimal solutions easily. Based on the GA-BP neural network, the fault prediction model of machine tool joint surface is established. With the help of this model, the fault after training can be predicted and the maintenance plan can be optmized.
\end{abstract}

\section{Introduction}

During the manufacturing process, the performance of each part of computer numerical control (CNC) machine tools declines. It causes the fault of $\mathrm{CNC}$ machine tools and reduces the production efficiency and product precision seriously. There are a large number of joint surfaces in the mechanical structure. They destroy the continuity of CNC machine tool and result in a complicated machine mode [1-3]. 40\% to $60 \%$ of the mechanical structures overall flexibility and over $90 \%$ of the total damping are derived from the joint surface. More than $60 \%$ of the vibration problems are connected to the joint surface [4]. According to statistics, due to mechanical component failure, China's annual losses of up to 200 billion Yuan (RMB). The maintenance costs of up to several hundred million Yuan (RMB).

For the safety and reliability of CNC machine tools, the downtime must be avoid and the processing costs cause from the fault of machine tools must be reduced. In recent years, the scholars from around the world have conducted a lot of researches on the fault diagnosis and the maintenance methods of machine tools [5-10]. The regular maintenance and the replacement of parts are usually used to shorten the maintenance cycle and reduce the maintenance time. According to these, the maintenance costs of the machine tool are controlled.

\section{Intelligent Maintenance Modelling of Moving Joints}

In order to design the intelligent maintenance of the slide guide, the vibration signal, the pressure signal, the temperature signal and the speed signal are monitored as the state data of the slide guide. The state data are processed to extract the eigenvalues, which can reflect the state of the guide joint surface. In this paper, the GA-BP neural network is used to fit the complex mapping between the signal characteristic value and the component state. In the real state, the mathematical model of $\mathrm{NC}$ machine tool is set up to realize the fault prediction of the key parts from machine tool. According to the results of the fault prediction and the traditional maintenance plan of the processing enterprises, the active maintenance scheme is formulated which is shown as Fig. 1. 


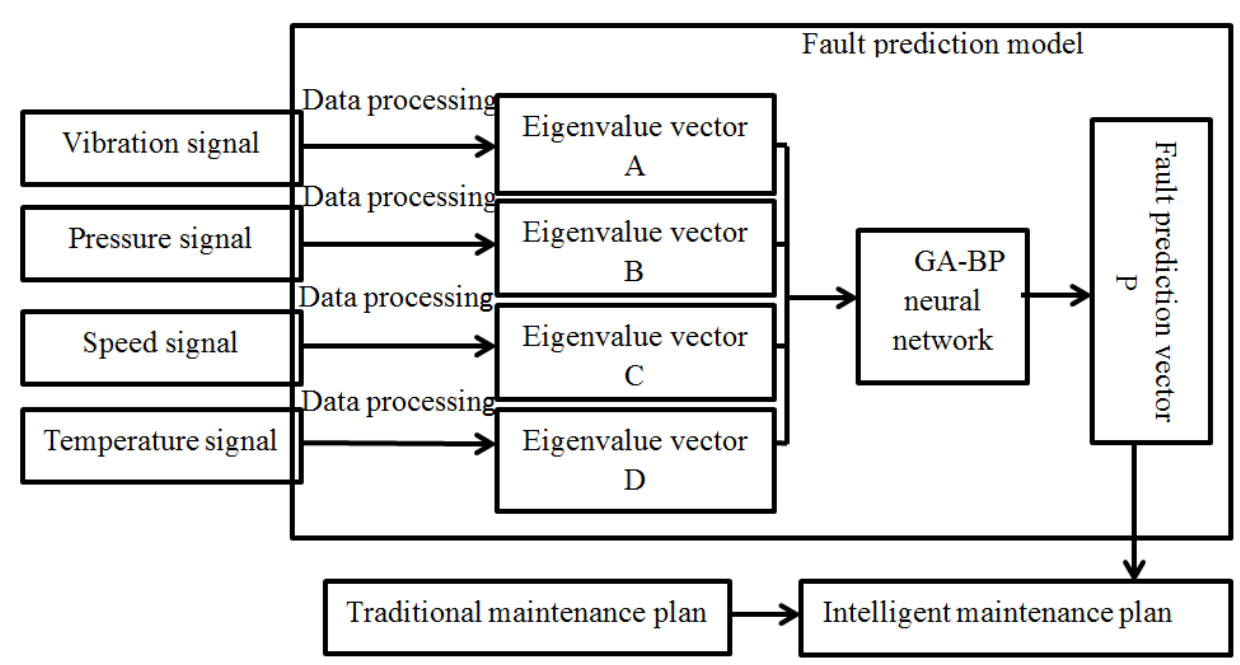

Figure 1. Fault prediction and intelligent maintenance

\section{Data Processing of Slide Guide.}

The vibration signal, pressure signal, temperature signal and speed signal of the guide rail are all disturbed. In order to reflect the state of the guide rail joint accurately, the signal is filtered. According to the signal characteristics of the key components of the machine tool, the signal is pre-treated by the band-pass filter, and the interference is eliminated.

In order to obtain the fault information of the joint, the sampling frequency of the vibration signal is $25 \mathrm{kHz}$, the pressure signal and the speed signal are all $10 \mathrm{kHz}$. Due to the high sampling frequency, it brings the huge amount of data. In order to improve the processing speed of fault prediction model, the data must be simplified. So the data grid cluster simplification is used.

Grid clustering is the method which can divide the data space into finite element (cell) grid structure. The data processing is based on a unit. The advantage of cluster processing is the high-speed processing. At the same time, the processing speed of the data is not directly related to the number of records in the target database, but only with the number of units in the data space.

According to the vibration data of the 65536 groups of machine tool guide rail with the grid clustering processing, in the range of error allowed, the adjustment of the cluster domain value is $0.005 \mathrm{~V}$, the data is simplified to 16382 groups. The vibration data before and after the simplified model is used to calculate the data samples of the fault prediction model, which makes the calculation time reduced by about $75 \%$. The simplified data samples can ensure the accuracy of the system. Fig. 2 shows the distribution of the original data.

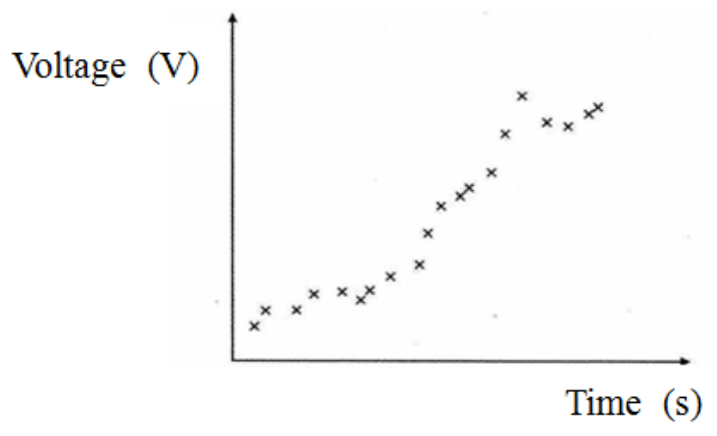

Figure 2. Distribution of the original data

Simplified data processed through the Fourier transform (FFT) extract the characteristic vector of signal, where the characteristic vector of vibration signal is $\mathrm{A}$, the characteristic vector of the pressure signal is $\mathrm{B}$, the characteristic vector of temperature signal is $\mathrm{C}$, the characteristic vector of the velocity signal is D. The four groups of the vectors are the input parameters of the artificial neural network, the weights of the neural network training. The neural network output vector $\mathrm{P}$ on behalf of the state of the 
guide joint surface, as shown in Table 1.

Table 1 Vector valued and state table

\begin{tabular}{|c|c|}
\hline $\mathrm{P}$ & State of joint surface \\
\hline 1 & Well \\
\hline 2 & Slight fault \\
\hline 3 & General fault \\
\hline 4 & Serious fault \\
\hline
\end{tabular}

\section{Fault prediction algorithm model.}

A fault prediction model is established by using BP+GA algorithm. The BP neural network technology is used in the dynamic characteristic study of the guide joint surface and the building of the guide joint surface. Genetic algorithm is used to train the initial weights of the network. The optimization results are used as the initial value of the BP neural network.

Fault prediction model algorithm is shown as Fig.3. Firstly, the weights (threshold) are coded by the floating point code. A set of distribution is generated randomly, which corresponds to a set of connection weights of neural networks (threshold value). Secondly, entering the training samples and calculating the error function value. In this function, the error square and its reciprocal is the degree of the adaption. If the error is smaller, the degree of adaptation will be better. On the contrary, it will be smaller. On this account, the connection right (threshold) can be evaluated. After the choice of a large degree of individual adaptation, inheriting to the next generation directly, and then using the crossover, mutation and other operations on the current population evolution, resulting in the next generation of groups. Repeating the above training method, so that a set of weights (threshold) has been evolving, until the training objectives meet the conditions. At last, the prediction samples are fed into the trained GA-BP neural network to predict the failure and the degree of the guide joint surface.

Selection, crossover and mutation operation are carried out to produce sub population.

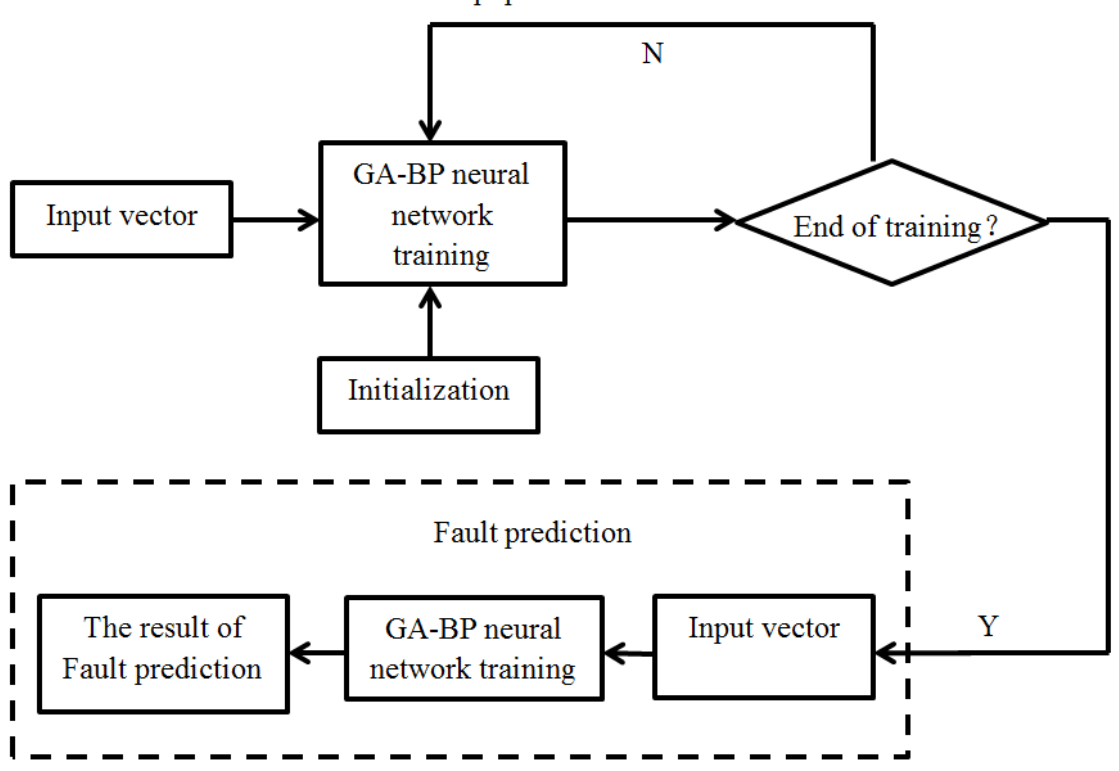

Figure 3. Fault prediction model algorithm

\section{Intelligent Maintenance.}

In order to develop the best maintenance plan, the spare parts plan and the maintenance program, the intelligent maintenance is based on CNC machine tools, components of the traditional repair methods and fault prediction results. The optimal maintenance plan can realize the shortest maintenance time of 
the CNC machine tool; reduce the machine down time and save the maintenance cost. The plans ensure that the parts can be replaced in a timely manner. At the same time, it can avoid a large number of the spare parts due to large amounts of funds. The optimal maintenance scheme can prolong the service life of the parts and machine tools, and delay the performance degradation of machine tools and components.

\section{BP+GA Algorithm Design of intelligent Maintenance Model}

Bp+Ga Algorithm. Both the genetic algorithm and the neural network are the research results of biological principles. The difference between them is that the biological evolution has a long process. Combining the two studies is to learn from the strengths of both, to find the effective ways to solve complex problems.

To optimize the initial weights and thresholds of BP neural network, it makes full use of the simple genetic algorithm, parallel processing structure and global optimal search advantages. Also, it overcomes the slow convergence speed of neural network and easy to fall into local extremum. It is unable to get to the disadvantages of the best weight distribution. After obtaining the initial weights and thresholds, self-study algorithm using BP neural network nonlinear approximation on the guide joint surface with dynamic characteristic parameters of the network model training, enables the network to achieve the expected requirements.

\section{Design on BP+GA Algorithm Model of Guide Joint Surface.}

Modelling of BP Neural Network. Material, surface roughness, sliding speed and temperature are the key factors that influence the surface properties of the joint surface. In this paper, the conventional three-layer BP neural network model is used, the above-mentioned five kinds of factors is selected as 5 input layers and output layers using two neurons (bonding surface tangential stiffness and normal stiffness).

In the hidden layer, 10 neurons are used to realize the whole connection in each layer. The Sigmoid function is used as the activation function of each neuron. The model structure is shown as Fig.4.

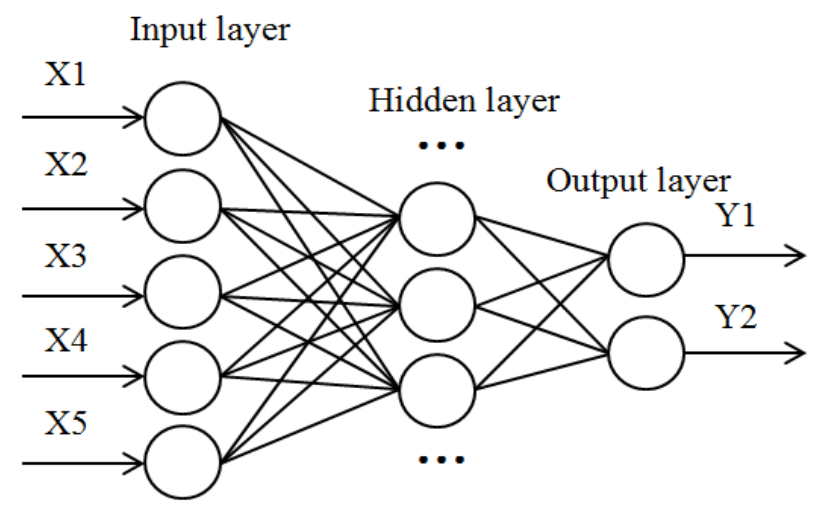

Figure 4. Extension and complement structure of neural network

Parameter Design of Genetic Algorithm. Coding is to combine the neural network weights in a certain way, and the GA algorithm is obtained. Neural network weights value learning is a complex continuous parameter optimization problem, right system is large, so the real number coding, neural network weights value according to a certain sequence for each position of a long string, string length corresponds to the network weights or thresholds.

The search target of GA algorithm is to make the network error square and the minimum network weight threshold in all the evolution generations. The GA algorithm can only move in the direction of the fitness function value. So we can calculate the square sum of the error of the BP network can be calculated according to the neural network generated by the weight threshold. 


\section{Conclusions}

In this paper, the joint surface fault prediction model is established by the GA-BP neural network to predict the fault of guide joint surface. Based on the advantages of GA-BP neural network (short training time, fast convergence, high precision and not easy to fall into local extremum), it has a good application prospect in the study of the dynamic characteristics of the joint surface. The grid clustering method is adopted to simplify the data and improve the calculation speed of the fault prediction of the joint surface. According to the fault prediction results, the optimal maintenance and spare parts plan are defined. It can reduce the maintenance costs and the machine downtime effectively. It can also improve the production efficiency obviously. So this method has a very wide range of engineering application value.

\section{Acknowledgements}

This work is supported by the National Natural Science Foundation of China, Grant No. 51405220, the Natural Science Foundation of Jiangsu Province, Grant No. BK20140764, the University Natural Science Research Project of Jiangsu Province, Grant No. 12KJA460002 and the Research Foundation of Nanjing Institute of Technology, Grant No. ZKJ201309.

\section{References}

[1] Z. Xue Liang, Dynamic characteristics and application of mechanical bonding surface, Beijing: China Science and Technology Press, (2002).

[2] Y. Ren, F. Beards, Identification of effective linear joints using coupling and joint identification techniques, ASME Journal of Vibration and Acoustics, 120(2) 331-338 (1998).

[3] R.A. Ibrahim, C.L. Pettit, Uncertainties and dynamic problems of bolted joints and other fasteners Vibration, 279(3) 857-936 (2005).

[4] Z. Guang Peng, H. Yu Mei, S. Wen Hao, Analysis method and application of dynamic characteristic of machine tool guide joint. J. Journal of Mechanical Engineering, 38 (10) 114-117 (2002).

[5] J. I. Park, B. Suk Joo, Direct prediction methods on lifetime distribution of organic light-emitting diodes accelerated degradation tests, IEEE Transactions on Reliability, 59(1) 74-90 (2010).

[6] G. Hong Li, L. Deng Wan, X. Ming Heng, Intelligent monitoring system for screw life evaluation, Journal of Southwest Jiaotong University, 45(5) 685-691 (2010).

[7] Z. Min, G. Hong Li, X. Ming Heng, Application of multi-variable grey model for ball screw remaining life prediction, Computer Integrated Manufacturing Systems, 17(4) 846-851 (2011).

[8] Z. Ying Zhi, J. Ya Zhou, S. Gui Xiang, Gray correlative analys is of failure distribution of CNC machine tool , Transactions of the Chinese Society of Agricultural Machinery, 35(6) 195-197 (2004).

[9] Z. Xue Zhi, Y. Bang Yan, C. Tong Jian, Difference spectrum theory of singular value and its application to the fault diagnosis of headstock of lathe, Journal of Mechanical Engineering, 46(1) 100-108 (2010).

[10] W. Wu, T. San, Z. Yuan Min. Prediction of surface roughness of high speed machining based on neural networks, Machinery Design and Manufacture, (3) 216-217 (2010). 